\title{
MENSURAÇÃO DE METABÓLITOS DE CORTISOL NAS FEZES COMO UM INDICADOR DE ESTRESSE EM BOVINO DE CORTE
}

\author{
MEASUREMENT OF CORTISOL METABOLITES IN FECES AS A STRESS INDICATOR \\ IN BEEF CATTLE
}

\begin{abstract}
Mobiglia, A.M. ${ }^{1 *}$; Camilo, F.R. ${ }^{1}$ e Fernandes, J.J.R. ${ }^{1}$
${ }^{1}$ Programa de Pós-Graduação em Produção Animal. Universidade Federal de Goiás. Escola de Veterinária e Zootecnia. Goiânia-GO. Brasil. *mellodeia@gmail.com
\end{abstract}

\section{Palavras chave adicionais}

Atividade adrenal. Bem-estar.

\section{RESUMO}

O estresse causado pelo manejo, transporte e outros agentes estressores, é uma rotina em sistemas de produção de bovinos de corte. Esse estresse pode gerar impacto econômico devido à redução da produção e da qualidade do produto final, além de ameaçar o bem-estar do animal que está inserido neste sistema. A situação de desconforto faz com que ocorram modificações fisiológicas, morfológicas e comportamentais aos animais. As alterações endócrinas e metabólicas são resultados dessa tentativa de manter a homeostase. Portanto, compreender os hormônios envolvidos na situação de estresse (atividade eixo hipotálamo-hipófise-adrenal) e seus metabólitos nos fluídos e nas excretas é uma forma de detectarmos o estresse animal. $O$ método invasivo, como a concentração de cortisol no plasma sanguineo é uma forma de monitoramento do estresse bastante questionada, uma vez que, esse hormônio aparece na corrente sanguinea logo após a situação de estresse e desaparece em curto prazo, de modo que o manejo para a coleta de sangue pode ser estressante ao animal o que provavelmente pode mascarar os resultados. Nessa linha de pensamento, surgiu a necessitade de estabelecer métodos não invasivos para monitorar a atividade adrenal em resposta ao estresse, como por exemplo, a mensuração da concentração de metabólitos de cortisol nas fezes de animais.

\section{SUMMARY}

The stress occurs by management, transport

\author{
Additional KeYWORdS \\ Adrenal activity. Welfare.
}

and other stressor agents that are routine in the production of beef cattle. This stress can impact in economics of system for decreasing production and quality of final product, in addition to threaten the animal welfare. The uncomfortable situation causes physiology, morphology and behavior modifications. When the animal tries to maintain homeostasis endocrine and metabolic changes occur. Therefore, understanding the hormones involved in a stress situation (hypothalamuspituitary-adrenal activity) and their metabolites in body fluids and excreta, can help to detect animal stress. The invasive methods, as the plasma cortisol concentration, to monitor the stress are very questioned, this hormone appears in blood after a stress situation and changes quickly. The management of animal to collect the blood may be stressful and can give a false result. By this reasons, it could be necessary to establish a noinvasive method to monitor the adrenal activity in response to stress, as could be the mensuration of concentration of cortisol metabolites in feces.

\section{ESTRESSE ANIMAL}

O estreses é definido como a ruptura da da homeostase dos organismos vivos em que existe uma resposta comportamental ou fisiológica contra estímulo nocivo ou condição adversa do ambiente (Maziero et al., 2012).

Os diversos sistemas de produção existentes para bovinos propiciam situações ou 


\section{MOBIGLIA, CAMILO E FERNANDES}

alterações ambientais, das quais os animais necessitam se adaptar através de modificações morfológicas, fisiológicas ou comportamentais.

O termo estresse tem sido definido como um estado que ocorre quando um animal necessita fazer ajustes extremos e anormais tanto em termos fisiológicos como comportamentais a fim de lidar com os aspectos negativos do ambiente e manejo (Fraser et al., 1975). A resposta fisiologica dos animais aos fatores estressantes vai induzir a secreção do hormonio liberador de corticotropina pelo hipotalomo o que ocorrera a secreção de corticotropina ou hormônio adrenocorticotrópico pela hipófise anterior. O hormônio adrenocorticotrópico age no córtex adrenal, que sintetiza e libera glicocorticóides no sangue, o animal quando recebe algum estimulo de estresse ativar o eixo hipotálamo-pituitário-adrenal (HPA), que vai aumentar os níveis plasmáticos do cortisol e corticoesterona (Kaneko et al., 1997).

A interação entre animal-homem é necessária para realizar o manejo nutricional, sanitário e reprodutivo, devendo ocorrer de forma mais positiva possível, uma vez que os bovinos aprendem com as experiências negativas, criando aversões a elas (Krohn et al., 2001). Outra situação estressante imposta aos bovinos durante sua vida é o transporte que compromete o bem-estar do animal e gera impactos econômicos (Palme et al., 2000), uma vez que o estresse causado pelo manejo pré-abate conduz a uma redução do $\mathrm{pH}$ do músculo post-mortem, originando uma carne indesejada como a carne DFD (dark, firm and dry) (Delezie et al., 2007). Essas alterações geram mudanças no status metabólico e endócrino do animal para que o mesmo se adapte aquela condição imposta.

$\mathrm{O}$ estresse também pode afetar diretamente a reprodução, pois em resposta ao estimulo de estresse ocorrera uma secreção hormonal pela glândula adrenal, a qual é controlada pelo hormônio liberador de corticotrofinas, que vai inibir a liberação de hormônios gonadotróficos pela adenohipófise, inbindo assim o pico préovulatório de LH ou inibir a ovulação e até mesmo piorar a qualidade dos embriões produzidos (Macedo et al., 2011).

O bem-estar animal é discutido não somente pelos cientistas, mas também pela sociedade em geral (Möstl e Palme, 2002). O indicador de bem-estar animal é a ausência de estresse animal, contudo, esta é uma questão que gera indagações, uma vez que não estão estabelecidos padrões de estresse e um único ensaio químico para mensurar o estresse (Hofer e East, 1998). Portanto, cada vez mais se torna necessário acrescentar parâmetros químicos e endócrinos para detectar esse distúrbio.

\section{METODOLOGIAS PARAMENSURAR O ESTRESSE ANIMAL}

Tanto o comportamento animal como a fisiologia estão envolvidos na resposta do animal ao estresse e comumente esses parâmetros são utilizados para avaliar o estresse animal (Broom e Johnson, 1993).

O comportamento animal é avaliado por meio de observações que requerem diversos recursos, como por exemplo: uma equipe treinada, a qual tenha o conhecimento especifico da espécie em questão, de forma que a análise do comportamento seja realizada criteriosamente com base no objetivo a ser alcançado ou então, a análise comportamental pode ser avaliada por meio de filmagens arquivadas provenientes de equipamentos de câmeras de vídeo capazes de obter filmagens durante o período diurno e noturno (Stewart et al., 2005).

Essa avaliação apesar de ser capaz de detectar alterações no comportamento não deve ser interpretada sem outros parâmetros, pois fatores como características individuais do animal, espécie, raça, experiência prévia do animal e o tipo de agente estressor podem influenciar no comportamento animal o que pode causar falha na interpretação e resul- 
tados subjetivos (Clark et al., 1997; Möstl e Palme, 2002).

O monitoramento de respostas fisiológicas ao estresse como a atividade hipotálamohipófise-adrenal através de alterações nos níveis de cortisol do plasma é outro parâmetro freqüentemente usado que sustenta os estudos de respostas ao estresse em animais de produção (Broom e Johnson, 1993). Por outro lado, existem limitações no uso desse hormônio para mensurar o estresse.

Dentre as dificuldades destacam-se o fato de não ser conhecido o nível padrão do hormônio no sangue das espécies, a freqüência de amostragem inadequada que podem perder de forma rápida as respostas do hormônio, além de ter sua maior limitação que é a necessidade da amostra de sangue do animal gerando um estresse de manejo para a obtenção da mesma que pode mascarar os resultados e até gerar interpretações errôneas (Stewart et al., 2005).

O nível de cortisol também pode ser afetado por outros fatores como status social (Mulleder et al., 2003), estado reprodutivo (Bell et al., 1991) e nível de atividade (Fisher et al., 2002). Grande diferenças na alimentação também podem alterar os níveis de metabólitos de cortisol nas fezes (Dantzer etal., 2011)

Outros meios de avaliar o estresse é pela mensuração do batimento cardíaco, taxa respiratória, temperatura corporal e nível de catecolamina (adrenalina) no sangue (Broom e Johnson, 1993). Porém esses dados não devem ser considerados isoladamente para mensurar o estresse. De acordo com Rushen et al. (2011) a combinação de diferentes mensurações (fisiológicas e comportamentais) devem ser consideradas para avaliar o estresse.

Em conclusão, não há um único e simples método para avaliar o estresse animal. Segundo Clark et al. (1997), são evidente a necessidade de um método confiável e não invasivo para mensurar o estresse de forma que seja possível considerar as respostas comportamentais e fisiológicas na obtenção dos resultados.

Alternativamente, têm se pesquisado procedimentos de amostragem não invasivos como a mensuração de metabólicos de cortisol nas fezes (Möstl et al., 2002; Palme et al., 2000), na urina (Hay e Morméde, 1998), saliva (Cooper et al.,1989) e no leite (Verkerk et al., 1998). Entretanto alguns desses métodos requerem manejo do animal para amostragem como a urina e a saliva. Com isso, os estudos de mensuração da concentração de metabólitos de cortisol nas fezes têm se destacado entre os métodos não invasivos de monitorar o estresse. A vantagem desse método é que as amostras podem ser coletadas de maneira fácil e possibilita repetidas mensurações por individuo (Touma e Palme, 2005).

\section{MECANISMOSFISIOLÓGICOSDO ESTRESSE ANIMAL}

A utilização de métodos não invasivos para monitorar a atividade adrenocortical requer o conhecimento do metabolismo e excreção do cortisol (glicocorticóides).

A descoberta de hormônios que estão relacionados com o estresse tornou possível descrever a reação do estresse em termos fisiológicos (Balm, 1999). Diversos hormônios estão envolvidos nesse processo fisiológico, tais como: hormônio adrecorticotrópico (ACTH), glicocorticóides (cortisol), catecolaminas (adrenalina e noroadrenalina), etc. (Matteri et al., 2000).

É através de estímulos externos do ambiente e hormônio que o eixo hipotálamohipófise-adrenal também chamado de eixo do estresse inicia a síntese do cortisol (Sheba et al., 2012).

Em uma situação estressante ao animal, informações sensoriais do ambiente são transmitidas para o sistema nervoso central (SNC) e por meio de impulsos nervosos estimula o hipotálamo a produzir hormônios hipotalâmicos. O hormônio hipotalâmico liberador de corticotrofina regula na hipófise 
anterior a secreção de um hormônio específico (ACTH) no sangue (Lehninger, 1985).

O ACTH é um polipeptídio de 39 aminoácidos e com meia vida de apenas dez minutos, sendo este hormônio sintetizado continuamente e destruído em um equilíbrio dinâmico (Lehninger, 1985). O hormônio específico (ACTH) se liga em receptores na superfície das células do córtex adrenal, mais especificamente na zona fasciculada, estimulando a produzir hormônios esteróides, como o cortisol (Glew, 2007).

Estes esteróides são chamados coletivamente de corticóides e são agrupados em três classes principais: os glicocorticóides (cortisol), mineralcorticóides (aldosterona) e corticosterona. Embora todos os hormônios esteróides sejam estruturalmente relacionados e bioquimicamente derivados do colesterol, eles possuem propriedades fisiológicas muito diferentes (Lehninger, 1985).

Os glicocorticóides, dos quais o cortisol é o mais importante, promove a neoglicogênese a partir dos aminoácidos e do depósito de glicogênio do fígado, aumentando a glicose sanguínea e redução da utilização de glicose periférica. O cortisol também estimula a utilização dos ácidos graxos e a cetogenêse, além de possuir ação antiinflamatória e anti-alérgica (Lehninger, 1985).

$\mathrm{O}$ animal exposto ao agente estressor por longo período fará com que ocorra a secreção excessiva de cortisol, levando o animal à fadiga e perda de massa muscular devido ao excesso de aminoácidos convertidos em glicose e da redistribuição da gordura no organismo (Lehninger, 1985).

\section{SÍNTESE DO CORTISOL NA CÉLULA FASCICULADADA ADRENAL}

O colesterol é precursor do cortisol, via pregnolona e é sintetizado na zona fasciculada da adrenal por esta região expressar a enzima $17 \alpha$-hidroxilase, que é necessária para formar a molécula de cortisol (Schimidt e Litwack, 2007).
O ACTH liberado pela hipófise se liga ao receptor de hormônio localizado na membrana celular de células fasciculadas da adrenal. Ativa-se a adenilato ciclase (AC) via proteína $\mathrm{G}$ estimulatória que juntamente com uma molécula de trifosfato de adenosina (ATP) faz com que o monofosfato de adenosina cíclico (cAMP) ative a proteína quinase A (PKA). A PKA por meio de fosforização aumenta a hidrolise de colesteril ésteres de vesícula a colesterol livre, além de aumentar o transporte do colesterol livre para dentro da mitocôndria por meio da proteína StAR que funciona entre as membranas mitocondriais externas e internas (Schimidt e Litwack, 2007).

O colesterol livre na mitocôndria é modificado pela enzima de clivagem de cadeia lateral (SCC) em pregnenolona (Granner, 2007), e a partir dessa molécula é sintetizado o cortisol que é secretado para fora da célula, ocorrendo o transporte para o sangue, onde irá atingir as células-alvo de glicocorticóides para expressar sua ação hormonal (Schimidt e Litwack, 2007).

Para síntese do cortisol requer três hidroxilases que atuam seqüencialmente nas posições $\mathrm{C}_{17}, \mathrm{C}_{21}$ e $\mathrm{C}_{11}$. A enzima $17 \alpha$ hidroxilase atua sobre a pregnenolona ou progesterona para formar a 17-hidroxipregnenolona (ou 17-hidroxiprogesterona). Essa molécula é hidroxilada (enzima 21hidroxilase) no $\mathrm{C}_{21}$ para formar o 11desoxicortisol, que em seguida é hidroxilado (enzima $11 \beta$-hidroxilase) no $\mathrm{C}_{11}$ para geraro cortisol (Granner, 2007).

A esteroidogênese requer idas e vindas repetidas do substrato para dentro e para fora da mitocôndria, através da proteína StAR pelo fato da enzima 21-hidroxilase estar presente no retículo endoplasmático liso, enquanto a enzima $11 \beta$-hidroxilase atua na mitocôndria (Granner, 2007).

\section{EXCREÇÃODOCORTISOLNAS FEZES}

O primeiro estudo de cortisol nas fezes 
foi realizado por Lindner em 1972 que infundiu ${ }^{14} \mathrm{C}$-cortisol na veia jugular de ovelhas e investigou a excreção do hormônio. Ele constatou que dois-terço da quantidade infundida foi subseqüentemente encontrado na bile. Os principais metabólitos encontrados foram glucoronato, tetrahidrocortisole e tetrahidrocortisona. Esses metabólitos de cortisol foram eliminados via fezes e urina.

A máxima concentração de metabólitos de cortisol encontrado nas fezes após a infusão de cortisol foi diferente entre várias espécies. Em ovelhas o pico de metabólitos de cortisol nas fezes foi obtido 12 horas após a infusão; em pôneis após 24 horas e em suínos obteve-se o pico depois de 48 horas (Möstl e Palme, 2002). Contudo, a excreção de cortisol é diferente para as espécies devido ao tempo de passagem intestinal de cada espécie.

Möstl et al. (2002) mostrou que 21 metabólitos de cortisol podem ser detectados em amostras fecais de ovelhas usando um cromatografo líquido de alta performance (espectrografia de massa). Entretanto, a rota metabólica e as enzimas que participam desse processo ainda são desconhecidas.

\section{TÉCNICASPARAMENSURAR METABÓLITOS DE CORTISOLNAS FEZES}

Palme e Möstl (1997) com o objetivo de validar uma técnica eficiente para mensurar os metabólitos de cortisol nas fezes de ruminantes testaram três enzimas (11oxoetiocholanolone; cortisol-3-CMO e corticosterone-3-CMO) para a técnica de teste imunoenszimático (enzyme immunoassay, EIA). Os autores concluíram que a enzima específica melhor para detectar os metabólitos derivados do cortisol foi a 11 oxoetiocholanolone EIA, técnica chamada pelos autores de 11,17-dioxoandrostane assay.

Palme et al. (1996) descreve que são esperados excreções de metabólitos por algum tempo após a infusão na veia. Somente a 11-oxoetiocholanolone-EIA, permitiu detectar os metabólitos com grandes picos e por mais dias após a infusão de cortisol. Os cortisol-EIA e corticosteroneEIA apenas apresentaram pequenos picos logo após a infusão, que segundo Palme e Möstl (1997) não foram eficaz para a técnica, concluindo que 11-oxoetiocholanoloneEIA (11,17-dioxoandrostane assay) pode ser utilizada como ferramenta para monitorar a atividade da adrenal em ruminantes.

Comercialmente há disponível imunoensaio de cortisol e corticosterona (ICN corticosterone RIA, MP Biomedicals, Costa Mesa-CA, USA) para mensurar os metabólitos de cortisol nas fezes validado para animais de produção domésticos (Palme, 2012).

\section{METODOLOGIAPARAMENSURAR METABÓLITOS DE CORTISOLNAS FEZES}

A metodologia foi estabelecida por Palme e Möstl em 1997, pesquisadores da University of Veterinary Medicine, Vienna, Austria, sendo o último protocolo da técnica lançado em 2010 (dados fornecidos pelo autor).

\section{Coletadas amostras}

As fezes devem ser coletadas logo após a defecação e congeladas imediatamente (<30 min) $\mathrm{a}-20^{\circ} \mathrm{C}$ até as análises, a fim de evitar a degradação dos metabólitos por enzimas.

Estudo com elefantes africanos (Loxodonta africana) em que se mensuraram os metabólitos de progesterona nas fezes foi encontrado maior concentração do hormônio quando realizou-se a subamostragem dentro da massa fecal, enquanto que a amostragem superficial nas fezes obteve menores concentrações de progesterona. Com base nisso o autor recomendou a homogeneização da massa fecal antes da amostragem para análises (Wasser et al., 
1996). Millspauch et al.(2001) e Millspauch e Washburn (2004) recomendaram que em estudos com mensuração de metabólitos de cortisol também deve-se homogeneizar a massa fecal e retirar uma sub-amostragem para análises, pois os metabólitos de cortisol nas fezes não estão distribuídos uniformemente.

\section{EXTRAÇÃODOSMETABÓLITOSDECORTISOLE PREPARAÇÃO DA AMOSTRA PARA ANÁLISE}

Devem ser pesados 0,5 gramas de fezes e adicionado $5 \mathrm{~mL}$ de metanol a $80 \%$ (prémistura de $4 \mathrm{~mL}$ de $100 \%$ metanol e $1 \mathrm{~mL}$ de água).

Estudos mostraram que a diluição de $80 \%$ de metanol foi mais eficaz em preservar os metabólitos de cortisol nas fezes Palme e Möstl (1997).

A extração utilizando $80 \%$ de metanol foi a mais eficiente segundo o autor, porém a extração $70 \%$ e $90 \%$ obtiveram o mesmo resultado uma vez que ambas não diferiram estatisticamente $(\mathrm{p}>0,05)$ da extração com $80 \%$ de metanol.

Após a extração com metanol (80\%) essa amostra deve ser agitada em centrifuga (2500 g;15 minutos), agitador manual (handvortex, 1 a 2 minutos) ou no agitador automático (multivortex, por 30 minutos). $\mathrm{O}$ sobrenadante deve ser pipetado e congelado a $-20{ }^{\circ} \mathrm{C}$ até a análise pelo método de imunoensaio enzimático (EIA).

A técnica com ensaio imunoenzimático é a mais usada para este tipo de análise, embora a caracterização dos metabólitos seja feia através de cromatografo líquido de alta performance (espectrografia de massa) como descrito em detalhes por Möstl et al. (2005).

\section{Resultados das PESQUISAS UTILIZANDO ATÉCNICA}

Experimento conduzido com vacas leiteiras $(n=5)$ que receberam ACTH sintético através de cateter na jugular comparou as concentrações de cortisol no plasma com as concentrações de metabólitos de cortisol nas fezes. O cateter foi colocado no dia $1 \mathrm{e}$ amostras de sangue foram coletadas nos dias 1 e 2 às 9 horas. No dia 3 foram coletadas amostras de sangue após a infusão de ACTH ( $9 \mathrm{~h} \mathrm{e} 11 \mathrm{~h}$ ) a cada 30 minutos das 8:30 h as 15:00 h (6,5 horas). Adicionalmente, amostras de sangue foram coletadas $12 \mathrm{~h}$, $24 \mathrm{~h}$ e $48 \mathrm{~h}$ e as amostras de fezes foram coletadas 8 a 45 h (em toda defecação espontânea) após a primeira dose de ACTH. A concentração basal de cortisol no plasma foi de 5,2 $\pm 0,8 \mathrm{ng} / \mathrm{mL}$. Observou-se maiores picos de cortisol $(40 \pm 3 \mathrm{ng} / \mathrm{mL} ; \mathrm{p}<0,01)$ no sangue 30 minutos após a primeira dose recebida de ACTH. O nível de concentração basal retornou após terminar a sessão de coleta de sangue de 6,5 horas. Evidenciando assim o método de coleta de sangue sendo invasivo quando monitora-se a concentração de cortisol no sangue para detectar o estresse. Por outro lado, a concentração de metabólitos de cortisol nas fezes teve resultados diferentes quanto ao pico desse hormônio após a dose de ACTH. O nível basal para a concentração de metabólitos de cortisol nas fezes foi de 567,5 ng/gramas de fezes e após 8 horas observou-se o primeiro pico, porém o maior pico $(3565 \pm 188,2 \mathrm{ng}$ /gramas de fezes; $\mathrm{p}<0,001)$ detectado foi entre 14 e 18 horas após a primeira dose de ACTH (Morrow et al., 2002).

Um segundo experimento realizado por Morrow et al. (2002), utilizou 8 vacas leiteiras da raça Holandesa que foram transportadas por $7,8 \mathrm{~km}$ (17 minutos) em caminhão boiadeiro. Os animais ao chegarem no destino permaneceram em piquetes ( 2 animais/ piquete) com acesso livre a água e comida por seis horas. Ao final do dia eles retornaram a fazenda de origem. Foram coletadas amostras fecais antes do transporte e $6 \mathrm{~h}$ (T6), 24 h (T24) e $30 \mathrm{~h}$ (T30) após o primeiro transporte. A concentração de metabólitos de cortisol nas fezes desses animais aumentou $159,8 \pm 15,1 \%$ nas amostras coletadas no T6 $(18,09 \pm 0,71 \mathrm{ng} / \mathrm{g}$ fezes; $\mathrm{p}=$ $0,0019)$. A concentração retornou ao nível basal (11 $\pm 0,79 \mathrm{ng} / \mathrm{g}$ fezes) 24 horas após a 


\section{METABÓLITOS DE CORTISOL NAS FEZES COMO UM INDICADOR DE ESTRESSE}

primeirajornada.

Estudando também o efeito da concentração de metabólitos de cortisol nas fezes de bovinos após o estresse do transporte Palme et al. (2000) constatou que o transporte eleva a concentração de metabólitos de cortisol nas fezes devido ao estresse do animal. Os autores utilizaram 16 vacas e dividiram em três tratamentos: $\mathrm{T}$, transporte de 8 animais por 2 horas; $S$, transporte de 4 animais por duas horas, porém o caminhão permaneceu estacionado por 3 horas antes do retornar a fazenda; e o grupo C que eram 4 animais que permaneceram no local. Foram coletadas amostras antes de iniciar o experimento (transporte) e coletadas amostras em toda defecação espontânea dos animais por 48 horas. O grupo $\mathrm{C}$ manteve a concentração de metabólitos de cortisol nas fezes durante todo o experimento. Por outro lado, o grupo $\mathrm{T}$ e $\mathrm{S}$ tiveram variações significativas $(\mathrm{p}=0,001)$ após o transporte. O pico máximo dos metabólitos ocorreu 12 horas após iniciar o transporte $(\mathrm{T}=964 \mathrm{nmol} / \mathrm{kg}$ fezes $; \mathrm{S}=$ $407 \mathrm{nmol} / \mathrm{kg}$ fezes) e retornou os valores basais $(\mathrm{T}=88 \mathrm{nmol} / \mathrm{kg}$ fezes $; \mathrm{S}=99 \mathrm{nmol} / \mathrm{kg}$ fezes) após 26 a 48 horas do transporte.

Esses dados evidenciam que o transporte em animais (gado e cavalos) é um agente estressor com alto impacto na resposta fisiológica dos animais (Möstl et al., 2002; Schmidt et al., 2010). O atraso do aparecimento de picos de metabólitos nas fezes permite o monitoramento de distúrbios ocorridos por volta de 12 horas antes. Todavia, saber a quantidade e a duração da liberação de metabólitos de cortisol nas fezes é uma variável biologicamente importante (Sheriff et al., 2011).

Estudo conduzido com vacas $(n=207)$ comparou o efeito de duas diferentes formas de contenção para casqueamento nos níveis de metabólitos de cortisol nas fezes. Animais que foram casqueados em bretes móveis para casqueamento apresentaram significantemente maior concentração de metabólitos de cortisol nas fezes que animais que foram casqueados em brete de mesa inclinável. Portanto, o estresse é maior em vacas casqueadas com bretes móveis (Pesenhofer et al., 2006).

Tucker et al. (2007) e Webster et al. (2008) relataram que durante a estação de inverno, as vacas expostas ao clima frio e úmido permaneceram menos tempo deitadas e apresentaram maior concentração de metabólitos de cortisol nas fezes comparando quando esses animais foram mantidos em local coberto.

\section{CONSIDERAÇÕESFINAIS}

$\mathrm{O}$ estresse animal pode trazer impacto tanto para o ponto de vista de bem-estar animal como econômico. Em sistemas de produção, principalmente quando se trata de bovinos, é necessária a aproximação do ser humano com esses animais para manejos, além disso, em algum momento da vida desses animais eles serão transportados e essas situações podem ser consideradas estressantes.

Uma forma de responder ao agente estressor são alterações comportamentais e fisiológicas a fim de manter o equilíbrio térmico no animal. Para entender o impacto que essas respostas causam na produção é preciso compreender os mecanismos de funcionamento dos principais hormônios do estresse, como é o cortisol.

$\mathrm{O}$ método de mensuração de estresse como a concentração de cortisol no plasma sanguíneo pode ter resultados subjetivos, uma vez que esse hormônio tem resposta imediata ao agente estressor e a coleta de amostra de sangue é uma fonte de estresse ao animal.

Diante do exposto, o estabelecimento de métodos não invasivos como a mensuração da concentração de metabólitos de cortisol nas fezes é uma alternativa para monitorar a atividade adrenal sem interferir nos resultados. Contudo, os fatores que influenciam essa excreção de metabólitos de cortisol nas fezes ainda são desconhecidos. Necessitando entender mais detalhadamente 
o metabolismo e como estes aparecem em forma de metabólitos de cortisol nas fezes. Por mais que a metodologia para a determinação da concentração de metabólitos de cortisol nas fezes já esteja estabelecida e

\section{BIBLIOGRAFIA}

Balm, P.H.M. 1999. Stress physiology in animals. Sheffield Academic Press. Sheffield. England. 284 pp.

Bell, M.E.; Wood, C.E. and Keller-Wood, M. 1991. Influence of reproductive state on pituitaryadrenal activity in the ewe. Domest Anim Endocrin, 8: 245-254.

Broom, D. M. and Johnson, K.G. 1993. Stress and animal welfare. Chapman and Hall. United Kingdom.

Clark, J.D.; Ranger, D.R. and Calpin, J.P. 1997. Animal well-being. Lab Anim Sci, 47: 586-597.

Cooper, T.R.; Trunkfield, H.R.; Zanella, A.J. and Booth, W.D. 1989. An enzyme-linked immunosorbent assay for cortisol in the saliva of man and domestic farm animals. Endocrinology, 123: 13-26.

Dantzer, B.; McAdam, A.G.; Palme, R.; Humphries, M.M.; Boutin, S. and Boonstra, R. 2011. How does diet affect fecal steroid hormone metabolite concentrations? An experimental examination in red squirrels. Gen Com Endocr, 174: 124131.

Delezie, E.; Swennen, Q.; Buyse, J. and Decuypere, E. 2007. The effect of feed withdrawal and crating density in transit on metabolism and meat quality of broilers at slaughter weight. Poultry Sci, 86: 1414-1423.

Fisher, A.D.; Verkerk, G.A.; Morrow, C.J. and Matthews, L.R. 2002. The effects of feed restrivtion and lying deprivation on pituitaryadrenal axis regulation in lactating cows. Livest Prod Sci, 73: 255-263.

Fraser, D.; Ritchie, J.S. and Fraser, A.F. 1975. The term stress in a veterinary context. Brit Vet, 131: 653-662.

Glew, R.H. 2007. Metabolismo dos lipídeos II: vias do metabolismo de lipídeos especiais. In: Manual de bioquímica com correlações clinicas. $6^{\mathrm{a}}$ ed. Editora Bucher. São Paulo. pp. 684-725.

Granner, D.K. 2007. A diversidade do sistema bem aceita pelos pesquisadores, ainda há muito que se pesquisar para entender todas as variáveis que podem influenciar nesse método não invasivo de avaliação do estresse animal.

endócrino. In: Bioquímica ilustrada. $27^{\text {a }} \mathrm{ed}$. McGraw-Hill. Porto Alegre. pp. 411-430.

Hay, M. and Mormède, P. 1998. Urinary excretion of catecholamines, cortisol and their metabolites in Meishan and Large White sows: validation as a non-invasive and integrative assessment of adrenocortical and symphatoadrenal axis activity. Vet Res, 29: 119-128.

Hofer, H. and East, M.L. 1998. Biological conservation and stress. Adv Stud Behav, 27: 405-525.

Kaneko, J.J.; Harvey, J.W. and Bruss, M.L. 1997. Clinical biochemistry of domestic animals. $5^{\text {a }}$ ed. Academic Press. New York.

Krohn, C.C.; Jago, J.G. and Boivin, X. 2001. The effect of early handling on the socialization of young calves to humans. Appl Anim Behav Sci, 74: 121-123.

Lehninger, A.L. 1985. Hormônios. In: Princípios de bioquímica. $2^{\text {a }}$ ed. Editora Savier. São Paulo. pp. 513-536.

Macedo, G.G.; Zúccari, C.E.S.N.; Pinto de Abreu U.G.; Negrão, J.A. and Costa e Silva, E.V. 2011. Human-animal interaction, stress, and embryo production in Bos indicus embryo donors under tropical conditions. Trop Anim Health Prod, 43: 1175-82.

Matteri, R.L.; Carroll, J.A. and Dyer, C.J. 2000. Neuroendocrine responses to stress. In: The biology of animal stress. Cabi Publishing. Wallingford. UK. pp. 43-76.

Mazieiro, R.R.D.; Martin, I.; Mattos, M.C.C. e Ferreia, J.C.P. 2012. Avaliação das concentrações plasmáticas de cortisol e progesterona em vacas nelore (Bos taurus indicus) submetidas a manejo diário ou manejo semanal. Vet Zootec, 19: 366-372.

Millspaugh, J.J. and Washburn, B.E. 2004. Use of fecal glucocorticoid metabolite measures in conservation biology research: considerations for application and interpretation. Gen Comp

Archivos de zootecnia vol. 63 (R), p. 8. 


\section{METABÓLITOS DE CORTISOL NAS FEZES COMO UM INDICADOR DE ESTRESSE}

Endocr, 138: 189-199.

Millspaugh, J.J.; Woods, R.J.; Hunt, K.E.; Raedeke, K.J.; Brundige,G.C.; Washburn, B.E. and Wasser, S.K. 2001. Using fecal glucocorticoid assays to study the physiological stress response of elk. Wildlife Soc B, 29: 899-907.

Morrow, C.J.; Kolver, E.S.; Verkerk, G.A. and Matthews, L.R. 2002. Fecal glucocorticoid metabolites as a measure of adrenal activity in dairy cattle. Gen Comp Endocr, 126: 229-241.

Möstl, E. and Palme, R. 2002. Hormones as indicators of stress. Domest Anim Encocrin, 23: 67-74.

Möstl, E.; Maggs, J.L.; Schrötter, G.; Besenfelder, $U$. and Palme, R. 2002. Measurement of cortisol metabolites in faeces of ruminants. Vet Res Commun, 26: 127-139.

Möstl, E.; Rettenbacher, S. and Palme, R. 2005. Measurement of corticosterone metabolites in birds' droppings: an analytical approach. Ann NY Acad Sci, 1046: 17-34.

Mulleder, C.; Palme R.; Menke, C. and Waiblinger, S. 2003. Individual differences in behaviour and in adrenocortical activity in beef-suckler cows. Appl Anim Behav Sci, 84: 167-183.

Palme, R. 2012. Monitoring stress hormone metabolites as a useful, non-invasive tool for welfare assessment in farm animals. Anim Welfare, 21: 331-337.

Palme, R. and Möstl, E. 1997. Measurement of cortisol metabolites in faeces of sheep as a parameter of cortisol concentration in blood. Int J Mam Biol, 62: 192-197.

Palme, R.; Fischer, P.; Schildorfer, H. and Ismail, M.N. 1996. Excretion of infused ${ }^{14} \mathrm{C}$-steroid hormones via faeces end urine in domestic livestock. Anim Reprod Sci, 43: 43-63.

Palme, R.; Robia, C.; Baumgartner, W. and Möstl, E. 2000. Transport stress in cattle as reflected by an increase in faecal cortisol metabolites. Vet Rec, 146: 108-109.

Pesenhofer, G.; Palme, R.; Pesenhofer, R.M. and Kofler, J. 2006. Comparison of two methods of fixation during functional claw trimming, walkin crush versus tilt table, in dairy cows using faecal cortisol metabolite concentrations and daily milk yield as parameters. Wien Tierarztl Monat, 93: 288-294.

Rushen, J.; Butterworth, A. and Swanson, J.C. 2011. Animal behavior and well-being sympo- sium: farm animal welfare assurance: science and application. J Anim Sci, 89: 1219-1228.

Schimidt, T. e Litwack, G. 2007. Bioquímica de hormônios. In: Manual de bioquímica com correlações clinicas. $6^{a}$ ed. Editora Bucher. São Paulo. pp. 871-925.

Schmidt, A.; Möstl, E.; Wehnert, C.; Aurich, J.; Müller, J. and Aurich, C. 2010. Cortisol release and heart rate variabilty in horses during road transport of one, 3.5 and 8 hours duration. Horm Behav, 57: 209-215.

Sheba, M.J.M.; Priva, B.; Meenambigai, D. and Pulliyur, S.M. 2012. Environmental stress and amelioration in livestock production neuroendocrine regulation of adaptive mechanisms in livestock. Springer. Berlin Heidelberg. pp. 263298.

Sheriff, M.J.; Dantzer, B.; Delehanty, B.; Palme, R. and Boonstra, R. 2011. Measuring stress in wildlife: techniques for quantifying glucocorticoids. Oecologia, 166: 869-887.

Stewart, M.; Webster, A.L.; Cook, N.J. and Scoot, S.L. 2005. Infrared thermografy as a noninvasive tool to study animal welfare. Anim Welfare,14: 319-325.

Touma, C. and Palme, R. 2005. Measuring fecal glucocorticoid metabolites in mammals and birds: The importance of validation. Annals NY Acad. Sci, 1046: 54-74.

Tucker, C.B.; Rogers, A.R.; Verkerk, G.A.; Kendall, P.E.; Webster, J.R and Matthews, L.R. 2007. Effects of shelter and body condition on the behaviour and physiology of dairy cattle in winter. Appl Anim Behav Sci, 105: 1-13.

Verkerk, G.A.; Phipps, A.M.; Carragher, J.F.; Matthews, L.R. and Stelwagen, K. 1998. Characterization of milk cortisol concentrations as a measure of short-term stress responses in lactating dairy cows. Anim Welfare, 7: 77-86.

Wasser, S.K., Papageorge, S., Foley, C. and Brown, J.L. 1996. Excretory fate of estradiol and progesterone in the African elephant (Loxodonta africana) and patterns of fecal steroid concentrations throughout the estrous cycle. Gen Comp Endocr, 102: 255-262.

Webster, J.R.; Stewart, M.; Rogers, A.R. and Verkerk, G.A. 2008. Assessment of welfare from physiological and behavioural responses of New Zealand dairy cows exposed to cold and wet conditions. Anim Welfare, 17: 19-26. 